\title{
Labyrinthe
}

33 | 2009 (2)

"Patates chaudes " : poétique, savoirs, politique

\section{Hayden White, l'ironie de la Métahistoire / En avant l'après-histoire!}

David Schreiber et Marc Aymes

\section{(2) OpenEdition}

Édition électronique

URL : http://journals.openedition.org/labyrinthe/4028

DOI : $10.4000 /$ labyrinthe.4028

ISSN : 1950-6031

Éditeur

Hermann

Édition imprimée

Date de publication : 23 octobre 2009

Pagination : 13-19

ISBN : 978-2-7056-6976-8

\section{Référence électronique}

David Schreiber et Marc Aymes, « Hayden White, l'ironie de la Métahistoire / En avant l'après-histoire!

», Labyrinthe [En ligne], 33 | 2009 (2), mis en ligne le 23 octobre 2011, consulté le 19 avril 2019. URL : http://journals.openedition.org/labyrinthe/4028; DOI : 10.4000/labyrinthe.4028

Propriété intellectuelle 


\section{HAYDEN WHITE, L'IRONIE DE LA MÉTAHISTOIRE/ EN AVANT L'APRÈS-HISTOIRE !}

David Schreiber

Voici le premier texte de Hayden White L'ironie, oui: elle est là tout de traduit en français. Il s'agit de l'introduction de son ouvrage le plus célèbre, Metahistory: The Historical Imagination in nineteenthcentury Europe ${ }^{1}$, introduction qui constitue à elle seule une sorte de petit livre dans le grand. Depuis longtemps, Labyrinthe espérait contribuer à combler cette importante lacune : il faut remercier Hayden White de nous y avoir autorisé ainsi que Laurent Ferri d'avoir traduit ce texte dense et difficile, presque quarante ans après sa publication initiale. Depuis déjà longtemps, l'ouvrage entier a été traduit en italien, en espagnol, en allemand. La France ne manque enfin plus à l'appel. suite dans le titre. C'est d'abord un peu de persiflage: il y a bien eu la Métaphysique après la Physique, qu'attendez-vous pour ajouter la Métahistoire à l'Histoire ${ }^{2}$ ? Et puis la réappropriation par White du terme metahistory est à elle seule un coup de force, comme une antiphrase: depuis R.G. Collingwood, crédité pour son invention, le mot était jusque-là très péjoratif, trop connoté "philosophie de l'histoire ", parmi les historiens de profession ${ }^{3}$. Vouloir en retourner la polarité, du moins au plus, c'était manifestement titiller des susceptibilités.

Né en 1928, Hayden White a accompli toute sa carrière aux États-Unis; il fut en poste à partir de 1978 à l'université de Californie à Santa Cruz. À l'origine historien du Moyen Âge - sa thèse portait sur les conflits autour de la question de l'autorité pontificale aux XI ${ }^{\mathrm{e}}$ et XII ${ }^{\mathrm{e}}$ siècles -, Hayden White s'est lui-même toujours considéré d'abord comme un historien, bien que son enseignement fût pour une grande part dispensé dans des départements de littérature comparée ou de rhétoriquet. À Santa Cruz, en particulier, l'histoire qu'il professe n'est pas canonique: c'est « l'histoire de la conscience ", history of consciousness ${ }^{5}$. Très au fait des discussions épistémologiques des années 1950 et 1960 dans le monde anglo-saxon, à la suite de l'article célèbre de Carl G. Hempel publié en $1942^{6}$, Hayden White inscrit dès le départ sa réflexion dans la perspective des philosophies narrativistes de l'histoire. Metahistory en élargit fortement le cadre, en proposant une éblouissante construction théorique qui va bien au-delà de la réflexion épistémologique qui avait cours. Le livre s'accompagne assez vite de la publication de Tropics of discourse, un recueil d'articles publiés dans diverses revues entre le milieu des années 1960 et la fin des années 1970. Commencent alors les véritables débats sur les propositions théoriques de White, jusque-là un peu confidentielles. Un second recueil, The Content of the form, publié en 1987, amplifie encore les discussions, qui atteignent leur apogée au début des années 1990, au moment de la publication des actes du colloque « Nazism and the Final Solution: Probing the Limits of Representation », tenu à Los Angeles en avril 19907. Enfin, un dernier recueil, Figural Realism, paru en 1999, propose quelques-uns des très nombreux articles publiés par Hayden White jusqu'à cette date ${ }^{8}$. 


\section{Labyrinthe, $n^{\circ} 33$}

Il serait difficile de résumer en quelques Le problème c'est ce damné méta- dont lignes la somme des positions de White, la signification prolifère. Il est de toutes changeantes au fil des années. Rappelons les combines: l'après, le retrait, l'aubrièvement ce que furent les thèses princi- delà, l'arrière-monde, la réflexivité... pales de Metahistory: en considérant le texte Comme Ulysse, il est polytropos ${ }^{10}$. historique comme une «structure verbale Veut-on le neutraliser, le plus simple ayant la forme d'un discours narratif », White sera de dire: puisqu'il se croit si intelentendait remettre en question le «noble ligent, laissons-le divaguer dans son rêve » de l'objectivité historique, sur lequel univers parallèle, le pauvre n'a plus se fondait la profession académique 9 . C'est la structure verbale elle-même qui vient donner aux événements historiques non seulement leur signification mais aussi leur statut d'événement réel. Dès lors, il n'est pas nécessaire de prendre en compte la visée référentielle du texte historique pour en comprendre le fonctionnement et celui-ci ne diffère pas fondamentalement du texte de fiction ou même des grandes constructions philosophiques de l'histoire dont l'emphase stylistique - au sens que White donne au mot style - n'est qu'une forme parmi d'autres. Il n'y a donc aucune raison de séparer la réflexion sur l'écriture de l'histoire des modalités d'écriture de la aucun sens des réalités. C'est ainsi que post, variante chic d'après, est devenu suffisant pour jeter l'opprobre: Hou le post-moderne! Vise un peu le poststructuraliste!

C'est bien commode. Les « tournants», les lignes de partage des eaux-comme le watershed à la télévision, lorsque la soirée avance et que vient l'heure de débrider les programmes, pour laisser place au nu et au gros mot-il en faut. Metahistory, c'est cela, comprenezvous enfin? La débauche à heure de grande écoute.

fiction.

Alors oui, pourquoi pas? S'il s'agit de reconnaître la nature problématique du langage (comme d'autres pratiques signifiantes) dans le moment même de son usage: oui, il semble bien que Metahistory soit d'après le "tournant " qu'on a proclamé " linguistique ». S'il s'agit de prendre acte d'une textualisation de l' "objectivité » et de "l'effet de réel », d'un engouffrement de la critique littéraire dans le " document 》: oui, il faut aussi lui faire endosser le "post-moderne ». Il n'y a pas juste moi et l'objet d'étude, l'actif et le passif. Il y a aussi, comme dans certaines langues, la voix moyenne: j'agis et suis agi11. L'analyste est constitutivement analysé, empêtré dans ce qu'il analyse, aux prises avec les significations qu'il parle et qui le parlent ${ }^{12}$. 
L'analyse formelle, quasi structurale, qui Seulement voilà: la voix de White retient ouvre Metahistory, est suivie d'une série beaucoup d'autorité, d'actif. Michelet, de chapitres monographiques sur les prin- Ranke, Tocqueville, Burckhardt, Hegel, cipaux représentants de l'historiographie et Marx, Nietzsche, Croce: c'est d'eux de la philosophie de l'histoire du XIX ${ }^{\mathrm{e}}$ siècle: Michelet, Ranke, Tocqueville, Burckhardt, Hegel, Marx, Nietzsche et Croce. White expérimente avec eux la théorie du récit et la théorie des tropes édifiées dans le chapitre introductif. Parcourir ainsi ces grandes œuvres du XIX ${ }^{\mathrm{e}}$ siècle, c'était s'attaquer aux fondements même du savoir historique « moderne ». Rapprocher les histoires des philosophies de l'histoire et l'écriture de l'histoire de celle de la fiction, c'était faire fi des lignes rouges tracées par les historiens professionnels pour identifier le périmètre de la science historique. On pourrait dire en simplifiant que Tropics of the discourse est venu par la suite étendre la théorie des tropes et The Content of the form la théorie du récit. La référence à Barthes et au fameux texte sur « le discours de l'histoire ${ }^{13}$ » est devenue centrale après Metahistory et White a maintenu ses positions jusqu'aux limites, y compris devant les constructions historiques narratives sur les crimes du $\mathrm{xx}^{\mathrm{e}}$ siècle. L'insistance sur les soubassements politiques et idéologiques d'un récit historique, que l'on trouve déjà dans Metahistory, s'est faite plus forte et White a mis l'accent sur les implications morales en jeu dans la narration. En critiquant la position de Pierre Vidal-Naquet sur la vérité, la nonvérité et le mensonge des récits historiques sur le génocide nazi ${ }^{14}$, White se confrontait à ce qui offrait le plus de résistance à sa théorie. Sans nier bien évidemment l'existence de faits historiques et leur documentation, White soutenait, contre Vidal-Naquet, que « la méthode» de l'historien ne permet pas d'invalider une interprétation et d'en valider une autre - ici, un certain discours sioniste sur la fatalité du génocide dans le contexte diasporique européen. La "vérité » du récit est la conséquence d'un rapport de force et d'une « effectivité politique », et non de la simple cumulation des faits. qu'il est question, pas de Louis Gabriel Gauny ou Louis-François Pinagot, car ce sont des historiens et philosophes " canoniques, ceux qui font encore aujourd'hui figures de modèles reconnus pour l'historien » (p. 23). Assistant à cette procession, le débauché du méta- se pince. Il escomptait des philosophes plébéiens, un roman familial de la Révolution ou un grand massacre de chats; voici qu'on l'invite à s'incliner devant des White's dead males.

L'enjeu ici est celui de l'alliage entre tropologie et tropolitique. Car enfin les tropes ne prennent pas la parole tout seuls: il faut aussi, reconnaît(ra) White, approcher la rhétorique en tant que "théorie de la politique du discours ", déterminer de quels conflits celui-ci, en tant que parole autorisée, résulte ${ }^{15}$. Peut-être, à ce titre, Metahistory fut-il trop souvent réduit à sa plus simple expression: le "style " n'est pas seulement une affaire de langage, mais aussi d'idéologie (voir ici p. 43). Les tropes ne sont aucunement l'alpha et l'oméga de sa panoplie, comme une lecture tronquée semble l'avoir laissé supposer. Pour parer à cette obnubilation, les écrits de White après Metahistory éclipseront les tropes, au profit d'une " politics of interpretation $»^{16}$. 


\section{Labyrinthe, $n^{\circ} 33$}

D’une façon générale, il serait excessif de dire que Hayden White a été « reçu» par les historiens. La plupart ont assez vite souscrit à l'accusation de relativisme sceptique, revendiqué, et à ce qu'il mettait en question dans le travail de l'historien. Assez vite rangé parmi les tenants d'un linguistic turn, aussi vague que tentaculaire, White fut surtout lu et discuté au sein des études littéraires et par les philosophes; les quelques historiens qui ont cherché à intégrer ses propositions à leur travail ont d'ailleurs souvent été conduits à intégrer des départements d'études littéraires et à se trouver quelque peu en rupture de ban avec leurs confrères. La discussion s'est surtout faite dans des revues comme Critical Inquiry, Diacritics et surtout History and Theory, qui a consacré plus d'une vingtaine d'articles à Hayden White et un cahier spécial à Metahistory ${ }^{17}$. En France, l'ouvrage est contemporain de Comment on écrit l'histoire, de Paul Veyne et de «L'opération historiographique » de Michel de Certeau mais rien n'est dit sur White avant Temps et Récit de Paul Ricœur au début des années 1980. Aucune revue d'histoire n'a pris la peine de faire un compte rendu des travaux de White et les réactions d'indignation, cycliques, devant l'absence de traduction de Metahistory n'ont pas suffi à enclencher le processus. Est-ce à dire que le débat n'a pas eu lieu? Non, sans doute, car certains y ont participé, aux marges dans un premier temps, plus directement par la suite, avec la discussion des travaux de Ricœur ${ }^{18}$. Il y a cependant fort à parier que le rapport entre le nombre de références bibliographiques à Metahistory et le nombre de ses lecteurs réels soit anormalement et dangereusement élevé - serait-il supérieur à un qu'il y aurait déjà un problème - et que bon nombre n'en ont une connaissance que par ouï-dire.
Que White ait voulu fausser compagnie à ses collègues historiens, nul doute. Rien n'indique pourtant que son " going meta » soit assimilable à une embrassade du post-. Ne remarquez-vous pas en effet l'accent sur la "structure profonde de l'imaginaire historique", sur les "homologies structurales », et l'hommage à LéviStrauss et Jakobson (n. 13, p. 22, 52 n.1 p.54)? Ne voyez-vous pas que toute la démarche de Metahistory consiste à faire primer le code, le langage, en un mot la structure, sur la diachronie de l'histoire? Vingt-cinq ans plus tard, il n'en démord pas: "Mon livre Metahistory est fondamentalement structuraliste. [...] Je suis un structuraliste-ou plutôt: un structuraliste et un formaliste ${ }^{19}$."

Aux lèvres du métahistorien, donc, flotte le sourire de Lévi-Strauss: "Une histoire vraiment totale se neutraliserait elle-même: son produit serait égal à zéro ${ }^{20} »$. Tandis que tous battaient la campagne à la poursuite de son double post-moderne, lui élisait domicile dans le bocage d'un " modernisme » douillet, avec ses phases et ses grands auteurs ${ }^{21}$. Le méta- pourrait n'avoir été qu'un leurre, un fétiche destiné à choquer le bourgeois ${ }^{22}$. Au fond, l'après, il s'en moque.

"Peu importe comment on m'appelle. Les étiquettes n'ont aucune importance à mes yeux. Tout ce que j'ai à dire c'est: Ne vous souciez pas des étiquettes et des écoles. Tenez, voici un livre. Lisez-le. S'il vous aide dans votre travail à vous, tant mieux; sinon, passez à autre chose $e^{23}$. » 


\section{Hayden White, L'ironie de la Métahistoire/En avant L'après-histoire!}

Comment lire ce texte aujourd'hui? D'abord comme un monument dans le débat sur le récit et comme une audace théorique qui n'a plus guère court aujourd'hui, ni chez les historiens, ni chez les littéraires. Souhaitons que le lecteur trouve déjà là de quoi satisfaire un principe de plaisir. Il va de soi que presque tout a été dit sur la théorie des tropes, Hayden White ayant lui-même largement répondu - structure mentale profonde ou code rhétorique historiquement situé, règles intangibles de la constitution d'un récit historique ou lieu d'inventions et de reformalisations possibles, théorie structuraliste, pré-structuraliste ou post-structuraliste -, et il est peu probable qu'on gagne à rouvrir ce problème. Hayden White lui-même, dans le genre ironique voire provocateur qu'il affectionne, a pu déclarer: « Metahistory is passé ${ }^{25}$ ». Plus intéressant nous semble être le débat sur la « modernité », aussi bien dans les contours qu'Hayden White lui donne dans ses écrits que dans les tentatives faites ici et là pour s'y ancrer ou au contraire s'en extraire et rejoindre ce qui vient après - post-modernité, s'il faut absolument le désigner par des mots. Hayden White lui-même semble toujours se présenter comme un «moderniste », mais nombreux sont ceux qui l'emmènent aujourd'hui vers une historiographie post-moderne ${ }^{26}$. La question se pose aussi en ce qui concerne l'extension idéologique ou politique qu'il faut donner à la critique du récit ou à propos des formes de représentation littéraires qui pourraient être requises après les propositions modernistes. Hayden White a montré comment, parallèlement aux fondements narratifs de l'historiographie moderne, la littérature expérimentait d'autres formes d'écriture susceptibles de construire du sens et dont l'histoire pourrait s'inspirer. On pourrait enfin s'interroger davantage sur la plus ou moins grande synchronisation des contextes, du point de vue d'une histoire intellectuelle. Rien ne garantit en effet que la «modernité » historiographique en France prenne ses sources dans le même corpus que dans le monde anglo-saxon, comme le notait fort justement Bernard Lepetit ${ }^{27}$. En France, depuis le premier tiers du $\mathrm{Xx}^{\mathrm{e}}$ siècle, l'histoire a rompu les attaches qui la reliaient aux humanités pour venir se placer dans le giron des sciences sociales. Cela expliquerait en partie le cantonnement de la réception de White, trop loin du débat « épistémologique » jusqu'ici, et la difficulté à ancrer de façon convaincante le débat sur les liens avec la littérature ${ }^{28}$. Une fois éclaircies ces différences de contexte, on peut espérer que l'héritage de White sera fructueux; à l'heure où des colloques et des livres d'hommages sont organisés un peu partout dans le monde, commençons déjà par donner à cet héritage une chance d'exister.

1. Baltimore, Johns Hopkins University Press, 1973.

2. Pose aristotélicienne que redouble le titre de l'introduction, où s'annonce le mouvement vers la «Poétique».

3. Keith Jenkins, article « Metahistory » dans Kelly Boyd (dir.), Encyclopedia of historians and historical writing, Londres et Chicago, Fitzroy Dearborn Publishers, 1999, vol. 2, p. 805-806, ici p. 805.

4. Très récemment, Hayden White est revenu sur sa formation initiale. Voir l'entretien avec Erlend Rogne dans History and Theory, 48, février 2009, p. 63-75: http://www.historyandtheory.org/Rogne.pdf (visité le 11 juillet 2009).

5. Voir le site: http://histcon.ucsc.edu/ (visité le 10 juillet 2009).

6. «The Function of General Laws in History », Journal of Philosophy, 39, 1942, p. 35-48. La meilleure introduction à ces débats reste Paul Ricœur, Temps et récit, tome 1, Paris, Le Seuil, 1983, p. 200-301. 


\section{Labyrinthe, $n^{\circ} 33$}

7. Les actes de ce colloque ont été publiés par Saul Friedländer, Probing the Limits of representation, Cambridge, Harvard University Press, 1992.

8. Ces trois recueils, qui ne regroupent qu'une partie des nombreux articles et analyses publiés par White depuis les années 1960, furent édités aux presses de l'université John Hopkins. Signalons la très complète bibliographie mise en ligne par Ewa Domańska en 2007: http://www.staff.amu.edu.p1/ ewa/ Hayden_White_Bibliography.htm (visitée le 13 juillet 2009).

9. À propos de l'historiographie américaine des $\mathrm{XIX}^{\mathrm{e}}$ et $\mathrm{Xx}^{\mathrm{e}}$ siècles, voir Peter Novick, That Noble Dream: the "objectivity question » and the American historical profession, Cambridge, Cambridge University Press, 1988. Les pages 598-611 sont consacrées à la réception de Metahistory par les historiens professionnels.

10. $C f$. Hans Kellner, «A Bedrock of order: Hayden White's linguistic humanism », History and Theory, 19, 4, 1980, Beiheft 19: Metahistory: Six Critiques, p. 1-29, ici p. 1: «Polytropos semble être une épithète appropriée pour caractériser Hayden White, ainsi que polytropique pour son livre Metahistory» (nous traduisons).

11. Cf. Hayden White, «Writing in the Middle Voice ", Stanford Literature Review, 9, 2, automne 1992, p. 179-187.

12. À ce sujet voir Dominick LaCapra, « History, language, and reading: Waiting for Crillon », American Historical Review, 100/3, juin 1995, p. 799-828, ici p. 803-804.

13. Roland Barthes, «Le Discours de l'histoire », Informations sur les sciences sociales, 6, 4, 1967, repris dans Le Bruissement de la langue, Paris, Le Seuil, 1984, p. 153-174. Sur la référence à Barthes chez White, voir Carlo Ginzburg, « Just One Witness », dans Saul Friedländer, op. cit., p. 82-96. Le texte de Carlo Ginzburg a été récemment traduit en français: Un seul témoin, Paris, Bayard, 2007.

14. Voir « The Politics of historical interpretation: Discipline and de-Sublimation », Critical Inquiry, vol. 9, $\mathrm{n}^{\circ} 1,1982$, p. 113-137, repris dans The Content of the form, p. 58-83; « Historical Emplotment and the problem of truth », dans Saul Friedländer, op. cit., p. 37-53, repris dans Figural Realism, p. 27-43. 15. Entretien avec Hayden White dans Ewa Domańska, Encounters. Philosophy of history after postmodernism, Charlottesville et Londres, University Press of Virginia, 1998, p. 13-38, ici p. 20.

16. Elizabeth A. Clark, History, theory, text. Historians and the Linguistic Turn, Cambridge et Londres, Harvard University Press, 2004, p. 103. Voir aussi l'entretien avec Hans Kellner dans Ewa Domańska, op. cit., p. 56. Dans le même recueil d'entretiens, pourtant, White déclare réfléchir à un retour au « tropique », en tant que « continuum de la logique, de la dialectique et de la poétique (ibidem, p. 27-28). 17. Voir, en plus de Peter Novick, op. cit., l'excellent article, très complet mais malheureusement déjà ancien, de Richard T. Vann, "The Reception of Hayden White", History and Theory, vol. 37, n 2 , 1998, p. 143-161. Les prises de position contre Hayden White de la part des historiens professionnels anglo-saxons sont récurrentes, voir sur ce point Frank R. Ankersmit, « Hayden White's Appeal to the Historians ", History and Theory, vol. 37, 2, 1998, p. 182-193.

18. Voir plus particulièrement les articles de Roger Chartier et de Bernard Lepetit, publiés en 1993 dans un numéro spécial de la revue italienne Storia della Storiografia, 24: Roger Chartier, « Figures rhétoriques et représentations historiques », p. 133-142, repris dans Au Bord de la falaise, Paris, Albin Michel, 1998, p. 108-125; Bernard Lepetit, « L'historicité d'un modèle historiographique », p. 143-148, repris dans Carnets de croquis, Paris, Albin Michel, 1999, p. 80-87.

19. Entretien avec Ewa Domańska, op. cit., p. 19 et 27.

20. Claude Lévi-Strauss, La Pensée sauvage, Paris, Plon, 1962, p. 341.

21. Parfois, lassé, il a dû sortir de sa retraite, protester un peu: « Dans le Times Literary Supplement du 18 octobre 1992, un essai de la professeur Gertrude Himmelfarb s'en est pris à moi, parmi d'autres : à ses yeux, j'incarnais la conception post-moderniste de l'histoire.

- Ce n'est pas le cas?

- Mais non! [...] Je considère mon projet comme moderniste» (entretien avec Ewa Domańska, op. cit., p. 26). 


\section{Hayden White, L'ironie de la Métahistoire/En avant L'après-histoire!}

22. Ibidem: « Peu m'importe au fond si l'establishment des historiens s'en est offensé. Qu'ils soient choqués, c'était l'idée ».

23. Ibidem., p. 30.

24. Voir l'entretien avec Ewa Domańska, « Hayden White: the image of self-presentation », Diacritics, vol. $24, \mathrm{n}^{\circ} 1,1994$, p. 91-100.

25. Voir en particulier les travaux de Keith Jenkins, Re-thinking History, Londres et New York, Routledge, 1991, On "What is history? »: From Carr and Elton to Rorty and White, Londres et New York, Routledge, 1995, et l'ouvrage collectif qu'il a dirigé avec Sue Morgan et Alun Munslow, Manifestos for History, Londres et New York, Routledge, 2007, avec une conclusion de Hayden White. Je remercie Sabina Loriga d'avoir attiré mon attention sur cet auteur.

26. Voir en particulier « The Modernist Event», dans Figural Realism, op. cit., p. 66-86.

27. À quelques exceptions près; voir Jacques Rancière, Les Noms de l'histoire, Paris, Le Seuil, 1992, dont la traduction anglaise a été préfacée par... Hayden White : «Foreword: Rancière's revisionism », dans The Names of History: On the Poetics of knowledge (trad. par Hassan Melehy), Minneapolis, University of Minnesota Press, 1994, p. vii-xix.

28. Voir, parmi d'autres références, Kuisma Korhonen (dir.), Tropes for the past. Hayden White and the history / literature debate, Amsterdam / New York, Rodopi, 2006; Frank R. Ankersmit, Ewa Domańska, Hans Kellner (dir.), Re-figuring Hayden White, Stanford, Stanford University Press, 2009. Signalons la rencontre « Between History and Narrative : Colloquium in Honor of Hayden White » organisée les 24 et 25 avril 2009 à l'université de Rochester, et la publication annoncée pour 2010 d'un nouveau recueil de textes avec une introduction du maître d'œuvre, Robert Doran: The Fiction of narrative: Essays on history, literature and theory, 1957-2007, Baltimore, Johns Hopkins University Press. 